OPEN ACCESS

Edited by:

Justin R. Seymour,

University of Technology, Sydney,

Australia

Reviewed by:

Lisa Moore,

University of Southern Maine, USA

Michael Gillings,

Macquarie University, Australia

${ }^{*}$ Correspondence:

Hongna Zhang and Weishan Chang,

College of Animal Science

and Technology, Shandong

Agricultural University,

Daizong Street 61

Taian 271000, China

zhang186982@126.com;

weishanchang@126.com

Specialty section:

This article was submitted to Aquatic Microbiology, a section of the journal

Frontiers in Microbiology

Received: 31 January 2015 Accepted: 18 March 2015

Published: 31 March 2015

Citation:

Zhang $H$, Zhou Y, Guo S

and Chang W (2015) Multidrug resistance found in

extended-spectrum

beta-lactamase-producing Enterobacteriaceae from rural water

reservoirs in Guantao, China.

Front. Microbiol. 6:267.

doi: 10.3389/fmicb.2015.00267

\section{Multidrug resistance found in extended-spectrum beta-lactamase-producing Enterobacteriaceae from rural water reservoirs in Guantao, China}

\author{
Hongna Zhang ${ }^{1 *}$, Yufa Zhou ${ }^{2,3}$, Shuyuan Guo ${ }^{1}$ and Weishan Chang ${ }^{1 *}$ \\ ${ }^{1}$ College of Animal Science and Technology, Shandong Agricultural University, Taian, China, ${ }^{2}$ College of Animal Science \\ and Technology, Shanxi Agricultural University, Taigu, China, ${ }^{3}$ Animal Husbandry Bureau of Daiyue District, Taian, China
}

Extended-spectrum beta-lactamase (ESBL)-producing Enterobacteriaceae have been isolated from humans and animals across the world. However, data on prevalence of ESBL-producing Enterobacteriaceae from rural water reservoirs is limited. This study aimed to isolate and characterize ESBL-producing Enterobacteriaceae in rural water reservoirs in Guantao, China. ESBL-producing Enterobacteriaceae were found in 5 (16.7\%) of 30 sampled rural water reservoirs. Sixty-six individual isolates expressing an ESBL phenotype were obtained in the present study. Species identification showed that 42 representatives of Escherichia coli, 17 Klebsiella pneumoniae, 4 Raoultella planticola, and 3 Enterobacter cloacae. Twenty isolates contained a single bla gene, including CTX-M (17 strains), TEM (2 strains), and SHV (1 strain). Forty-six isolates contained more than one type of beta-lactamase genes. ESBL-producing Enterobacteriaceae isolated in this study were all multidrug resistant. These findings indicated that the serious contamination of ESBL-producing Enterobacteriaceae in rural water reservoirs existed in Guantao, China.

Keywords: ESBL, Enterobacteriaceae, rural water reservoirs, multidrug resistance, bla genes

\section{Introduction}

The rational use of antibiotics helps control infectious diseases of humans and animals. Abuse and overuse of antibiotics in clinical practice has selected drug resistant bacteria and "superbugs" (Thompson et al., 2007; Yong et al., 2009; Pruden et al., 2012). Extended-spectrum beta-lactamases (ESBLs), resulting from amino acid substitutions in TEM-1, TEM-2, and SHV-1 enzymes were described in the 1980s and 1990s (Bush and Jacoby, 2010). ESBLs can hydrolyze penicillins, oxyimino-cephalosporins (e.g., cefotaxime, ceftazidime, ceftriaxone, cefuroxime, cefepime) and aztreonam but not cephamycins (e.g., cefoxitin, cefotetan) or carbapenems (Bush and Jacoby, 2010; El Salabi et al., 2013). ESBLs are predominantly found among Enterobacteriaceae, which are inhabitants of intestinal flora and important pathogens in nosocomial and community settings (Laurent et al., 2008; Azap et al., 2010; Song et al., 2011; Kang et al., 2013).

Extended-spectrum beta-lactamase-producing Enterobacteriaceae can spread between humans via contaminated food or water (Oteo et al., 2010; Piednoir et al., 2011) and acquire resistance to antibiotics by plasmids, transposons or other mobile vectors that carry resistance elements (Oteo et al., 2010; Peirano et al., 2012). Water environments are considered as important reservoirs for 


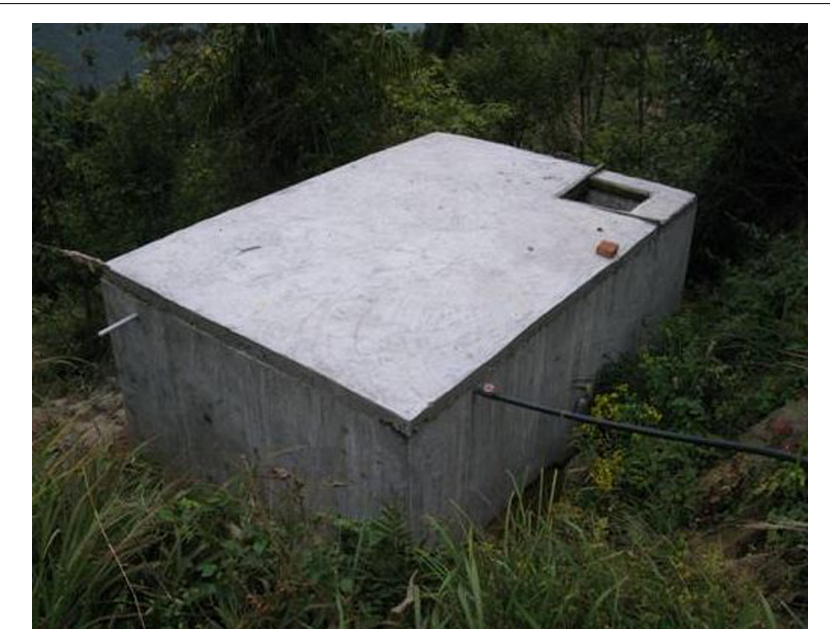

FIGURE 1 | The rural water reservoir commonly found in Guantao villages.

resistance genes (Gao et al., 2009), and maybe play an important role in transfer of drug-resistant genes between bacteria (Malakoff, 2002; Kummerer, 2004). More importantly, once ESBL-producing Enterobacteriaceae enter the intestine of humans and animals via drinking water, these bacteria could lead to the spread of resistance genes and to serious infections.

To date, numerous studies on ESBL-producing Enterobacteriaceae isolated from water environments have focused on wastewaters of hospitals and animal farms, and waters from rivers and lakes (Cabello et al., 2013; Varela and Manaia, 2013; Yang et al., 2013; Zurfluh et al., 2013; Haque et al., 2014). However, data on ESBL-producing Enterobacteriaceae isolated from drinking water in rural areas is very limited. In China, the main drinking sources for rural residents in many villages are water reservoirs. Therefore, the present study was conducted to describe the isolation and characterization of ESBL-producing Enterobacteriaceae in rural water reservoirs in Guantao, China.

\section{Materials and Methods}

\section{Sampling Sites and Water Sample Collection}

Between July and September of 2013, water sampling was conducted in Guantao, China (Figures 1 and 2). Five samples each were collected at six locations for a total of 30 samples. The water samples were collected from $50 \mathrm{~cm}$ below the water surface using sterile bottles (100 ml/bottle, one bottle/each reservoir). The collected water samples were stored on ice and immediately transported to our lab for further analyses within $3 \mathrm{~h}$.

\section{Microbiological Analysis}

Hundred milliliters of water was filtrated through a sterile $0.45 \mu \mathrm{m}$ membrane (Millipore, Billerica, MA, USA), and then the filters were incubated in $20 \mathrm{ml}$ of enterobacteria enrichment (EE) Broth (Becton Dickinson, Heidelberg, Germany) at $37^{\circ} \mathrm{C}$ for $24 \mathrm{~h}$. One loopful of enrichment cultures was spread onto chromogenic Brilliance ESBL agar (Oxoid, Hampshire, UK) and incubated at $37^{\circ} \mathrm{C}$ for $24 \mathrm{~h}$. The colonies with different color and morphology

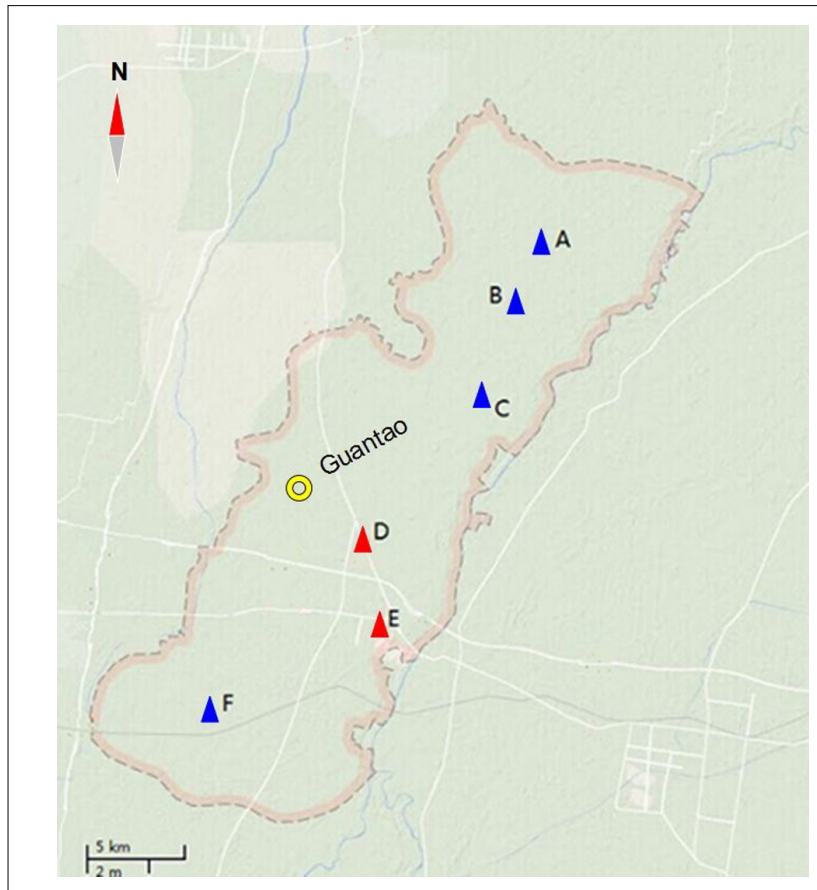

FIGURE 2 | Sampling sites. Blue triangles (A-C,F) represent the communities where no ESBL-producing Enterobacteriaceae were detected in water samples. Red triangles (D, E) represent the communities where ESBL-producing Enterobacteriaceae were found in water samples. Yellow circle represents urban areas of Guantao County.

were picked and sub-cultured on sheep blood agar for $24 \mathrm{~h}$ at $37^{\circ} \mathrm{C}$ (Huang et al., 2010). Conventional biochemical methods and API ID 32 E (bioMérieux, Marcy l'Etoile, France) were used to identify the isolates. If isolates showed doubtful results, they were subjected to genetic identification based on sequencing of $r p o B$ gene fragments (Mollet et al., 1997).

\section{Antimicrobial Susceptibility Testing and ESBL Confirmation}

According to the protocols of the Clinical and Laboratory Standards Institute (CLSI, 2011), the disk diffusion method was used to test susceptibility of the isolates against 17 antimicrobial agents. The tested antibiotics were: ampicillin (AMP), cefaclor (CEC), cefazolin (CFZ), cefepime (FEP), cefotaxime (CTX), ceftazidime (CAZ), ceftriaxone (CRO), cefuroxime (CXM), aztreonam (AZT), ciprofloxacin (CIP), gentamicin (GEN), imipenem (IPM), ofloxacin (OFX), piperacillin (PIP), amikacin (AMK), chloramphenicol (CHL) and tetracycline (TET). According to the manufacturer's protocols, Etest-ESBL strips (bioMérieux, Marcy l'Etoile, France) were used to confirm ESBL production. Isolates showing resistance to three or more antibiotic classes were defined as multidrug resistant (MDR). E. coli ATCC 25922 and K. pneumoniae ATCC 700603 were used as quality control strains.

\section{Polymerase Chain Reaction (PCR) to Detect bla Genes}

The DNA of the isolates confirmed for producing ESBLs was extracted separately using a DNA extraction kit (Biospin plasmid 
TABLE 1 | Species composition of ESBL-producing Enterobacteriaceae from water samples.

\begin{tabular}{lccc}
\hline Species composition & Number & Percent (\%) & Sources D/E \\
\hline E. coli & 42 & 63.6 & $18 / 24$ \\
K. pneumoniae & 17 & 25.8 & $7 / 10$ \\
Raoultella planticola & 4 & 6.1 & $2 / 2$ \\
Enterobacter cloacae & 3 & 4.5 & $1 / 2$ \\
Total & 66 & 100.0 & $28 / 38$ \\
\hline
\end{tabular}

extraction, Bioflux, Japan). According to previously published work, PCR was used to detect $b l a_{\mathrm{TEM}}, b l a_{\mathrm{CTX}-\mathrm{M}}$, and $b l a_{\mathrm{SHV}}$ genes using specific primers (Chen et al., 2010).

\section{Results}

\section{Detection of ESBL-producing Enterobacteriaceae}

Extended-spectrum beta-lactamase-producing Enterobacteriaceae were detected in five water reservoirs of two rural communities (D: 2, E: 3). The five water reservoirs were all located close to chicken farms (approximately 12-15 m). No ESBL-producing Enterobacteriaceae were found in the other reservoirs, which were far away from rural villages and animal farms (approximately 1.0-1.5 km).

Sixty-six different isolates exhibiting an ESBL phenotype were obtained (D: 28, E: 38). The results of species identification showed that 42 E. coli, 17 K. pneumoniae, 4 Raoultella planticola, and 3 Enterobacter cloacae (Table 1).

\section{Antibiotic Susceptibility of ESBL-producing Enterobacteriaceae}

Extended-spectrum beta-lactamase-producing Enterobacteriaceae isolates displayed similar drug-resistant trends. Nearly all ESBL-producing Enterobacteriaceae were resistant to the first- and second-generation cephalosporins (cefazolin, cefaclor, cefuroxime). These isolates were also resistant to the thirdgeneration cephalosporins: cefotaxime (91.5\%), ceftriaxone (67.9\%), and ceftazidime (31.1\%). Moreover, $48.1 \%$ of the isolates were resistant to cefepime (the fourth-generation cephalosporin), $51.9 \%$ to aztreonam (a monocyclic $\beta$-lactam antibiotic), and $89.6 \%$ to ampicillin.

Extended-spectrum beta-lactamase-producing Enterobacteriaceae were resistant to non- $\beta$-lactam antibiotics: resistant to ciprofloxacin (78.3\%), gentamicin (60.4\%), ofloxacin (76.4\%), piperacillin (68.9\%), chloramphenicol (55.7\%), and tetracycline (65.1\%). But the majority of ESBL-producing Enterobacteriaceae isolates were susceptible to amikacin (95.3\%) and imipenem (97.2\%) (Figure 3).

\section{Characterization of bla Genes in ESBL-producing Enterobacteriaceae}

All 66 ESBL-producing Enterobacteriaceae carried bla genes. Among 66 ESBL-carriers, 20 strains carried only one bla gene (20/66, 30.3\%), including 17 carrying $b l a_{\text {СTX-M }}(17 / 66,25.8 \%), 2$ isolates carrying bla $_{\mathrm{TEM}}(2 / 66,3.0 \%)$, and 1 carrying bla $_{\mathrm{SHV}}(1 / 66$, $1.5 \%)$. The other 46 isolates carried at least two bla genes (46/66,

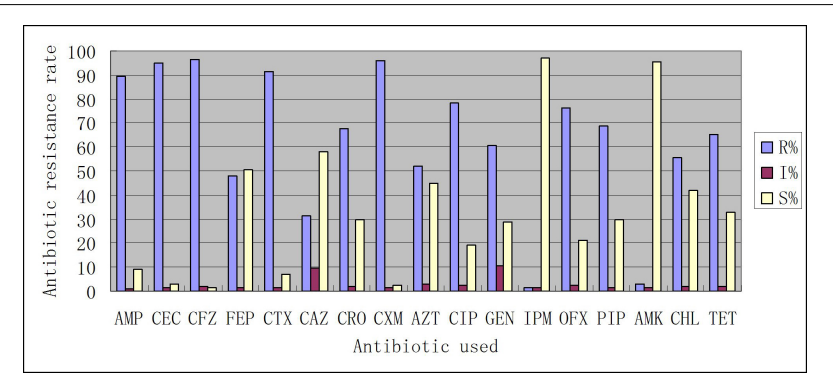

FIGURE 3 | Antibiotic resistance rates of ESBL-producing Enterobacteriaceae. R: Resistance; I: intermediate; S: susceptible; AMP, ampicillin; CEC, cefaclor; CFZ, cefazolin; FEP, cefepime; CTX, cefotaxime; CAZ, ceftazidime; CRO, ceftriaxone; CXM, cefuroxime; AZT, aztreonam; CIP, ciprofloxacin; GEN, gentamicin; IPM, imipenem; OFX, ofloxacin; PIP, piperacillin; AMK, amikacin; CHL, chloramphenicol; TET, tetracycline.

TABLE 2 | bla gene types of ESBL-producing Enterobacteriaceae from water samples.

\begin{tabular}{lccc}
\hline bla gene types & Number & Percent (\%) & Sources (D/E) \\
\hline TEM & 2 & 3.0 & $1 / 1$ \\
CTX-M & 17 & 25.8 & $6 / 11$ \\
SHV & 1 & 1.5 & $0 / 1$ \\
TEM+CTX-M & 38 & 57.6 & $18 / 20$ \\
SHV+CTX-M & 6 & 9.1 & $2 / 4$ \\
TEM+SHV+CTX-M & 2 & 3.0 & $1 / 1$ \\
Total & 66 & 100.0 & $28 / 38$
\end{tabular}

69.7\%), including 38 carrying $b a_{\mathrm{TEM}_{+} \mathrm{CTX}-\mathrm{M}}(38 / 66,57.6 \%), 6$ carrying $b a_{\mathrm{SHV}_{+} \mathrm{CTX}-\mathrm{M}}(6 / 66,9.1 \%)$, and the other 2 carrying three bla genes $(2 / 66,3.0 \%)$ (Table 2).

\section{Discussion}

In this study, ESBL-producing Enterobacteriaceae were detected in five out of 30 rural reservoirs $(5 / 30,16.7 \%)$, but not found in the other water reservoirs far away from villages and animal farms. This suggests that ESBL-producing Enterobacteriaceae are being shed into reservoirs from animal farms and anthropogenic activities.

Extended-spectrum beta-lactamase-producing Enterobacteriaceae isolates in this study were MDR. These isolates showed high resistance against the third-generation cephalosporins: cefotaxime $(91.5 \%)$, and ceftriaxone (67.9\%). Importantly, $48.1 \%$ of these isolates were resistant to cefepime, the fourth-generation cephalosporin. In addition, antibiotics resistance rates of these isolates to non- $\beta$-lactam antibiotics were also worrisome. But $95.3 \%$ and $97.2 \%$ of these isolates were respectively susceptible to amikacin and imipenem, which may be related with relatively low use of these medicines in this region.

In mainland China, previous investigations about ESBLproducing Enterobacteriaceae in water bodies and food-producing animals showed that $b l a_{\mathrm{CTX}-\mathrm{M}}$ gene was the dominant ESBL producer (Jin and Ling, 2006; Chen et al., 2010; Rao et al., 2014). Our data also identified $b l a_{\mathrm{CTX}-\mathrm{M}}$ gene as an important ESBL producer. Additionally, 46 out of 66 ESBL-producing Enterobacteriaceae isolates carried at least two bla genes and bla $a_{\text {СТХ-М }}$ TЕM 
has become the dominant phenotype of ESBL, which was different from the previous result in clinical isolates (Zhang et al., 2014).

There were some limitations in this study: water sampling was carried out only in 30 rural water reservoirs, so the results may not be representative of the whole area; sequence analyses of bla genes encoding TEM, CTX-M, and SHV were not further conducted

\section{References}

Azap, O. K., Arslan, H., Serefhanoğlu, K., Colakoğlu, S., Erdoğan, H., Timurkaynak, F., et al. (2010). Risk factors for extended-spectrum $\beta$-lactamase positivity in uropathogenic Escherichia coli isolated from community-acquired urinary tract infections. Clin. Microbiol. Infect. 16, 147-151. doi: 10.1111/j.1469-0691.2009. 02941.x

Bush, K., and Jacoby, G. A. (2010). Updated functional classification of $\beta$ lactamases. Antimicrob. Agents Chemother. 54, 969-976. doi: 10.1128/AAC. 01009-09

Cabello, F. C., Godfrey, H. P., Tomova, A., Ivanova, L., Dölz, H., Millanao, A., et al. (2013). Antimicrobial use in aquaculture re-examined: its relevance to antimicrobial resistance and to animal and human health. Environ. Microbiol. 15, 1917-1942. doi: 10.1111/1462-2920.12134

Chen, H., Shu, W. Q., Chang, X. S., Chen, J. A., Guo, Y. B., and Tan, Y. (2010). The profile of antibiotics resistance and integrons of extended-spectrum $\beta$ lactamase producing thermotolerant coliforms isolated from the Yangtze River basin in Chongqing. Environ. Pollut. 158, 2459-2464. doi: 10.1016/j.envpol.2010. 03.023

Clinical and Laboratory Standards Institute (CLSI). (2011). "Performance Standards for Antimicrobial Susceptibility Testing: Twenty-first Informational Supplement. Document M100-S21s. Wayne, PA: Clinical and Laboratory Standards Institute.

El Salabi, A., Walsh, T. R., and Chouchani, C. (2013). Extended spectrum $\beta$-lactamases, carbapenemases and mobile genetic elements responsible for antibiotics resistance in Gram-negative bacteria. Crit. Rev. Microbiol. 39, 113-122. doi: 10.3109/1040841X.2012.691870

Gao, P. P., Luo, Y., Zhou, Q. X., and Mao, D. Q. (2009). Research advancement of antibiotics resistance genes in aquaculture environment. Asian J. Ecotoxicol. 4, 770-779.

Haque, A., Yoshizumi, A., Saga, T., Ishii, Y., and Tateda, K. (2014). ESBL-producing Enterobacteriaceae in environmental water in Dhaka, Bangladesh. J. Infect. Chemother. 20, 735-737. doi: 10.1016/j.jiac.2014.07.003

Huang, T. D., Bogaerts, P., Berhin, C., Guisset, A., and Glupczynski, Y. (2010). Evaluation of Brilliance ESBL agar, a novel chromogenic medium for detection of extended-spectrum- $\beta$-lactamase-producing Enterobacteriaceae. J. Clin. Microbiol. 48, 2091-2096. doi: 10.1128/JCM.02342-09

Jin, Y., and Ling, J. M. (2006). CTX-M-producing Salmonella spp. in Hong Kong: an emerging problem. J. Med. Microbiol. 55, 1245-1250. doi: 10.1099/jmm.0. 46637-0

Kang, C. I., Wi, Y. M., Ko, K. S., Chung, D. R., Peck, K. R., Lee, N. Y., et al. (2013). Outcomes and risk factors for mortality in community-onset bacteremia caused by extended-spectrum $\beta$-lactamase-producing Escherichia coli, with a special emphasis on antimicrobial therapy. Scand. J. Infect. Dis. 45, 519-525. doi: $10.3109 / 00365548.2013 .775479$

Kummerer, K. (2004). Resistance in the environment. J. Antimicrob. Chemother. 54, 311-320. doi: 10.1093/jac/dkh325

Laurent, C., Rodriguez-Villalobos, H., Rost, F., Strale, H., Vincent, J. L., Deplano, A., et al. (2008). Intensive care unit outbreak of extended-spectrum $\beta$-lactamase-producing Klebsiella pneumoniae controlled by cohorting patients and reinforcing infection control Measures. Infect. Control Hosp. Epidemiol. 29, 517-524. doi: 10.1086/588004

Malakoff, D. (2002). Microbiologists on the trail of polluting bacteria. Science 29, 2352-2353. doi: 10.1126/science.295.5564.2352

Mollet, C., Drancourt, M., and Raoult, D. (1997). rpoB sequence analysis as a novel basis for bacterial identification. Mol. Microbiol. 26, 1005-10011. doi: 10.1046/j.1365-2958.1997.6382009.x to identify variants of these types of enzymes; the risk factors associated with rural reservoir water carriage of ESBL-producing Enterobacteriaceae were not further analyzed.

In summary, these findings indicated that the contamination of ESBL-producing Enterobacteriaceae in rural water environments existed in Guantao, China, and the pollution may be closely related to local animal farms and anthropogenic activities.

Oteo, J., Perez-Vazquez, M., and Campos, J. (2010). Extended-spectrum $\beta$-lactamase-producing Escherichia coli: changing epidemiology and clinical impact. Curr. Opin. Infect. Dis. 23, 320-326. doi: 10.1097/QCO. 0b013e3283398dc1

Peirano, G., van der Bij, A. K., Gregson, D. B., and Pitout, J. D. (2012). Molecular epidemiology over an 11-year period (2000-2010) of extended-spectrum $\beta$-lactamase-producing Escherichia coli causing bacteremia in a centralized Canadian region. J. Clin. Microbiol. 50, 294-299. doi: 10.1128/JCM.06025-11

Piednoir, E., Thibon, P., Borderan, G. C., Godde, F., Borgey, F., Le Coutour, X., et al. (2011). Long-term clinical and economic benefits associated with the management of a nosocomial outbreak resulting from extended-spectrum $\beta$ lactamase-producing Klebsiella pneumoniae. Crit. Care Med. 39, 2672-2677. doi: 10.1097/CCM.0b013e31822827e0

Pruden, A., Arabi, M., and Storteboom, H. N. (2012). Correlation between upstream human activities and riverine antibiotic resistance genes. Environ. Sci. Technol. 46, 11541-11549. doi: 10.1021/es302657r

Rao, L., Lv, L., Zeng, Z., Chen, S., He, D., Chen, X., et al. (2014). Increasing prevalence of extended-spectrum cephalosporin-resistant Escherichia coli in food animals and the diversity of CTX-M genotypes during 2003-2012. Vet. Microbiol. 172, 534-541. doi: 10.1016/j.vetmic.2014.06.013

Song, J. H., Thamlikitkul, V., and Hsueh, P. R. (2011). Clinical and economic burden of community-acquired pneumonia amongst adults in the Asia-Pacific region. Int. J. Antimicrob. Agents 38, 108-117. doi: 10.1016/j.ijantimicag.2011.02.017

Thompson, S. A., Maani, E. V., Lindell, A. H., King, C. J., and McArthur, J. V. (2007). Novel tetracycline resistance determinant isolated from an environmental strain of Serratia marcescens. Appl. Environ. Microbiol. 73, 2199-2206. doi: 10.1128/AEM.02511-06

Varela, A. R., and Manaia, C. M. (2013). Human health implications of clinically relevant bacteria in wastewater habitats. Environ. Sci. Pollut. Res. Int. 20, 3550-3569. doi: 10.1007/s11356-013-1594-0

Yang, F. X., Mao, D. Q., Luo, Y., Wang, Q., and Mu, Q. H. (2013). Horizontal transfer of antibiotic resistance genes in the environment. Chin. J. Appl. Ecol. 24, 2993-3002.

Yong, D., Toleman, M. A., Giske C. G., Cho, H. S., Sundman, K., Lee, K., et al. (2009). Characterization of a new metallo-beta-lactamase gene, $b / a_{(\mathrm{NDM}-1)}$, and a novel erythromycin esterase gene carried on a unique genetic structure in Klebsiella pneumoniae sequence type 14 from India. Antimicrob. Agents Chemother. 53, 5046-5054. doi: 10.1128/AAC.00774-09

Zhang, J., Zheng, B., Zhao, L., Wei, Z., Ji, J., Li, L., et al. (2014). Nationwide high prevalence of CTX-M and an increase of CTX-M-55 in Escherichia coli isolated from patients with community-onset infections in Chinese county hospitals. BMC Infect. Dis. 14:659-669. doi: 10.1186/s12879-014-0659-0

Zurfluh, K., Hächler, H., Nüesch-Inderbinen, M., and Stephan, R. (2013). Characteristics of extended-spectrum $\beta$-lactamase (ESBL)- and carbapenemaseproducing Enterobacteriaceae isolated from rivers and lakes in Switzerland. Appl. Environ. Microbiol. 79, 3021-3026. doi: 10.1128/AEM.00054-13

Conflict of Interest Statement: The authors declare that the research was conducted in the absence of any commercial or financial relationships that could be construed as a potential conflict of interest.

Copyright $\odot 2015$ Zhang, Zhou, Guo and Chang. This is an open-access article distributed under the terms of the Creative Commons Attribution License (CC BY). The use, distribution or reproduction in other forums is permitted, provided the original author(s) or licensor are credited and that the original publication in this journal is cited, in accordance with accepted academic practice. No use, distribution or reproduction is permitted which does not comply with these terms. 